\title{
EFECTOS ACÚSTICOS INMEDIATOS DE UNA SECUENCIA DE EJERCICIOS VOCALES CON TUBOS DE RESONANCIA
}

\section{Immediate effects of a vocal exercise sequence with resonance tubes}

\author{
Marco Guzmán ${ }^{(1)}$, Diego Higueras ${ }^{(2)}$, Catherine Fincheira ${ }^{(3)}$, Daniel Muñoz ${ }^{(4)}$, Carlos Guajardo ${ }^{(5)}$
}

\section{RESUMEN}

Objetivo: el propósito de este estudio es conocer el efecto acústico inmediato de una secuencia de ejercicios fonatorios utilizando tubos de resonancia en un grupo de sujetos disfónicos. Método: se aplicó una secuencia única de cuatro tareas fonatorias dentro de tubos de resonancia a veinticuatro profesores con voces disfónicas, evaluadas perceptualmente con la escala GRBAS. Las muestras de voz fueron grabadas antes y después de la secuencia de ejercicios. El análisis acústico incluyó Cepstrum, Jitter, Shimmer y relación armónico-ruido (NHR). Se solitó completar un protocolo de autovaloración vocal posterior a la secuencia de ejercicios para evaluar los efectos subjetivos. Se compararon los valores de los parámetros acústicos en las muestras pre y post entrenamiento. Resultados: cambios significativos fueron obtenidos en el análisis Cepstral, Jitter y relación armónico-ruido. En la autovaloración, los efectos positivos predominaron sobre los negativos, siendo los principales: voz más estable, voz más clara y garganta más abierta. Conclusión: el uso de tareas fonatorias dentro de tubos de resonancia puede tener un efecto fisiológico terapéutico inmediato en sujetos con voces disfónicas y una percepción subjetiva de mejoría en la producción de la voz posterior a la terapia.

DESCRITORES: Voz; Entrenamiento de la Voz; Acústica

\section{INTRODUCCIÓN}

Ejercicios vocales con oclusiones parciales o alargamiento del tracto vocal han sido ampliamente utilizados en la terapia y entrenamiento de la voz cantada $^{1}$ y hablada con el objetivo de producir un aumento en la impedancia de entrada del tracto vocal $^{2}$. Este tipo de ejercicios son producidos a través de estrechas constricciones en la parte anterior de la cavidad oral o en los labios. Algunas de estas técnicas incluyen el uso de consonantes

(1) Fonoaudiólogo; Profesor asistente Escuela de Fonoaudiología de la Universidad de Chile. Fundación Iberoamericana de voz cantada y hablada (FIVCH); Especialista en Vocología pela University of lowa.

(2) Fonoaudiólogo Universidad Austral de Chile.

(3) Fonoaudiólogo Universidad Austral de Chile.

(4) Medico Cirujano Universidad de Chile, Programa de Magister en Bioestadística da Facultad de Medicina de la Universidad de Chile.

(5) Fonoaudiólogo Universidad Nacional Andrés Bello; Docente Escuela de Fonoaudiología da Universidad Austral de Chile.

Conflito de interesses: inexistente oclusivas, consonantes fricativas, vibración de labios, lengua o fonación dentro de tubos de resonancia de diferente diámetro y longitud.

Los tubos de resonancia son utilizados de dos maneras: una forma es dejando el extremo distal del tubo libre en el aire y la otra forma es sumergiendo el extremo distal en agua. En ambos casos el tubo se mantiene derecho en la boca del sujeto, actuando como una extensión artificial del tracto vocal. La fonación dentro de tubos ha sido usada en la terapia vocal en Finlandia desde la década de los sesenta. Este tipo de ejercicios han sido utilizados también por personas con voces normales en entrenamiento y calentamiento de la voz ${ }^{3,4}$. En este último grupo se ha observado que cantantes logran una emisión más clara, brillante y resonante después del uso de tubos de resonancia ${ }^{5}$. Un beneficio ofrecido por los tubos de diámetro estrecho, que otros ejercicios como la vibración labial o lingual no ofrecen, es que se puede monitorear el sonido de la laringe de manera fácil por el hecho de no ser enmascarado por el sonido de las vibraciones de labios o lengua ${ }^{6}$. En el ámbito terapéutico, los tubos de resonancia han sido aplicados en 
casos de disfonía funcional (hiper e hipofuncional), en paresia unilateral de nervio laríngeo recurrente y en pacientes con nódulos entre otras patologías vocales ${ }^{7}$. El efecto fisiológico y acústico producido por los tubos puede variar dependiendo del largo del tubo utilizado ${ }^{8}$.

Uno de los efectos aerodinámicos producidos por el uso de tubos o estrechamientos del tracto vocal es el incremento del promedio de la presión supraglótica y por lo tanto un aumento de la presión intraglotal. Esto tiende a separar los pliegues vocales reduciendo el impacto mecánico al contactarse medialmente ${ }^{6}$. Otro efecto de la fonación dentro de tubos es el incremento de la reactancia positiva (inertancia) ${ }^{9}$. Story y Laukkanen ${ }^{10}$ demostraron que los cambios en la impedancia de entrada del tracto vocal son producidos por el uso se posturas semi-oclusivas o extensiones del tracto vocal. En este estudio el uso de extensiones del tracto vocal produjeron los valores de impedancia más altos después de la consonante bilabial oclusiva /b/. Titze ${ }^{9}$ demostró que la economía vocal puede mejorar al hacer coincidir la impedancia glotal con la impedancia del tracto vocal, especialmente si la reactancia del tracto vocal se mantiene positiva (inertiva) en el mayor rango de frecuencia fundamental posible. Desde el punto de vista acústico uno de los beneficios de los tubos de resonancia y oclusiones parciales del tracto vocal es el descenso del primer formante (F1), por lo tanto, la fonación de la frecuencia fundamental puede ser producida más fácilmente cerca de F1. Esto permitiría experimentar efectos de un bajo umbral de presión de la fonación, una disminución del flujo de aire transglótico y una voz rica en armónicos.

Algunos estudios sugieren que la impedancia de entrada del tracto vocal puede afectar el flujo glotal, la forma del pulso glótico y las características oscilatorias de los pliegues vocales ${ }^{11,12}$.En relación a lo anterior Titze y Story ${ }^{13}$ señalan que un incremento de la reactancia debido a un estrechamiento de la zona baja del tracto vocal puede descender el umbral fonatorio e incrementar el skeewing de la forma de flujo glótico (cesación más rápida del flujo), produciendo un fortalecimiento de los armónicos altos e incluso un incremento en el nivel de presión de sonido. Esto implicaría una producción más económica de la voz (mayor salida de sonido con menor estrés mecánico de los pliegues vocales). Titze ${ }^{6}$ estudió la fonación dentro de tubos estrechos, lo cual aumenta la resistencia supraglótica en forma considerablemente mayor a los tubos de resonancias con diámetros anchos. Una presión subglótica alta y signos de una colisión más suave entre los pliegues vocales fue observada durante la fonación en tubos en comparación con la fonación de vocales. El autor concluyó que con el uso de tubos de resonancia es posible producir grandes presiones subglóticas con una colisión mínima entre los pliegues vocales. Otros parámetros fonatorios como cambios en la frecuencia fundamental han sido reportados. Laukkanen ${ }^{14}$ en un estudio con tubos de diferentes largos, señaló que cuando se utilizó un tubo de plástico de $2.5 \mathrm{~cm}$. de diámetro se observó una caída en el valor de F0 en hombres al ser la longitud del tubo de $60 \mathrm{~cm}$. y en las mujeres cuando tenía $30 \mathrm{~cm}$. de largo.

El propósito de este estudio es conocer el efecto acústico inmediato de una secuencia de ejercicios fonatorios utilizando tubos de resonancia en un grupo de sujetos con voces perceptualmente disfónicas.

\section{MÉTODO}

\section{Evaluación}

La muestra estuvo compuesta por 24 profesores con edades entre 23 y 57 años, promedio de 36.45 años, portadores de voces disfónicas. El diagnóstico y grado de disfonía fue realizado perceptualmente por un fonoaudiólogo con 10 años de experiencia en el tratamiento de las alteraciones de la voz, utilizando la escala GRBAS. Todos los participantes fueron catalogados con el número 1 en el grado de disfonía (G) y grado de aspiración (S). 18 de los sujetos incluidos en el estudio fueron de sexo femenino y 6 de sexo masculino. Ninguno de ellos tenía antecedentes de terapia vocal previa a la realización de este estudio.

Se grabó la voz de cada sujeto en forma previa a la terapia y otra posterior a ésta. La grabación se realizó utilizando un micrófono de condensador marca Samson, modelo MM01. El micrófono fue puesto sobre un pedestal y situado a $10 \mathrm{~cm}$ de la boca de los sujetos y con un grado de inclinación de aproximadamente 90 grados. Los sujetos permanecieron sentados durante la grabación. Como sistema de pre amplificación y digitalización se utilizó la interfaz de sonido Tascam US-122L. Las grabaciones se realizaron con una frecuencia de muestreo de $22.050 \mathrm{~Hz}$ y 16 bit dentro de una cámara silente. La captura y grabación de las señales de voz fueron realizadas con el programa Goldwave V5.57 instalado en un computador portátil Sony Vaio Y-210.

Se grabó la vocal /a/ en forma mantenida durante la mayor cantidad de tiempo que cada sujeto pudiera sostener en una frecuencia fundamental e intensidad cómodas.

El análisis acústico incluyó la realización de Cepstrum, Jitter, Shimmer y relación armónicoruido $(\mathrm{NHR})$ de todas las muestra de voz tomadas 
pre y post tratamiento vocal. Para el análisis Cepstral se utilizó el software Lingwaves de la empresa Wevosys. La obtención de los valores de Jitter, Shimmer y NHR se realizó con el programa PRAAT versión 5.2. El análisis de Cepstrum fue realizado con una window sample de 1024 y una window type Hamming.

El valor de Cepstrum es una medida a corto plazo que se obtiene en un punto determinado de la forma de onda. Por esta razón se tomaron 6 puntos en la zona media de cada onda analizada (por tener mayor estabilidad) y luego los valores fueron promediados.

\section{Terapia}

El programa terapéutico aplicado en este estudio estuvo compuesto por una secuencia de 4 tareas fonatorias realizadas utilizando tubos de resonancia de $0.3 \mathrm{~cm}$. de diámetro interno y 22.8 de longitud.

Los ejercicios fonatorios fueron los siguientes:

1) Fonación en tubo produciendo una frecuencia mantenida y cómoda para cada sujeto en intensidad y altura. Figura 1

2) Fonación en tubo produciendo glissandos ascendentes y descendentes. Figura 2

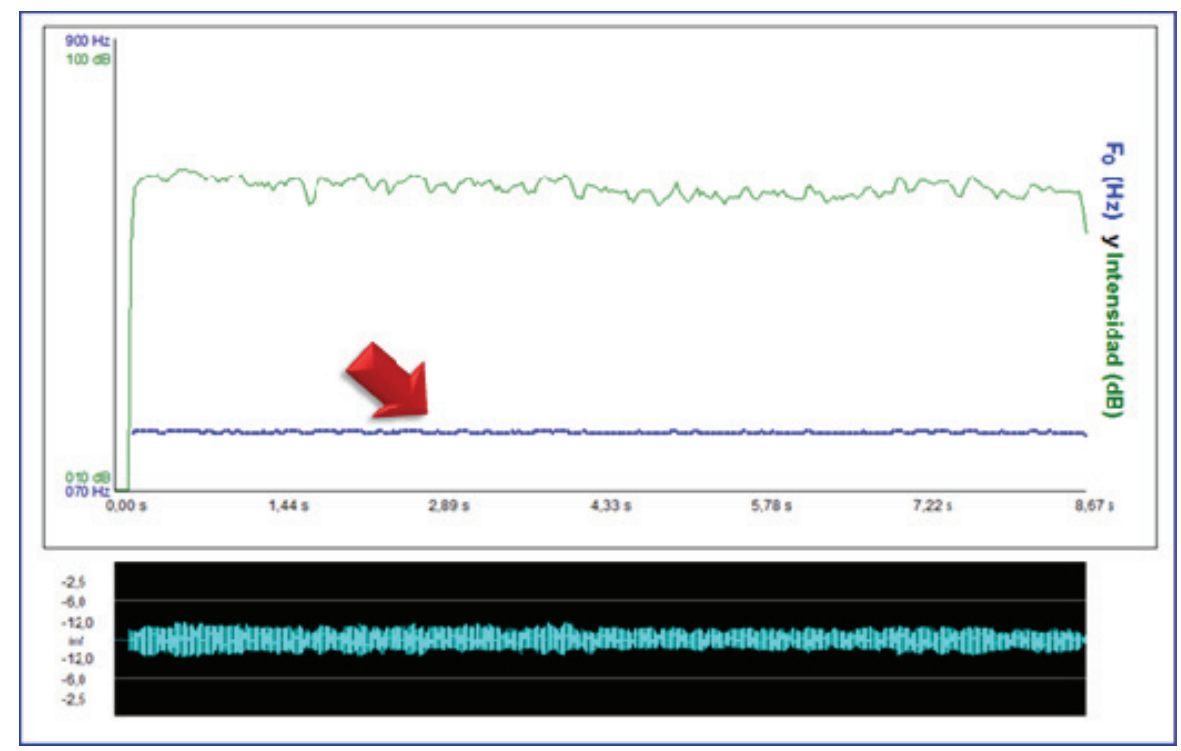

Figura 1 - Diagrama de fonación en tubo produciendo una frecuencia mantenida y cómoda para cada sujeto en intensidad y altura

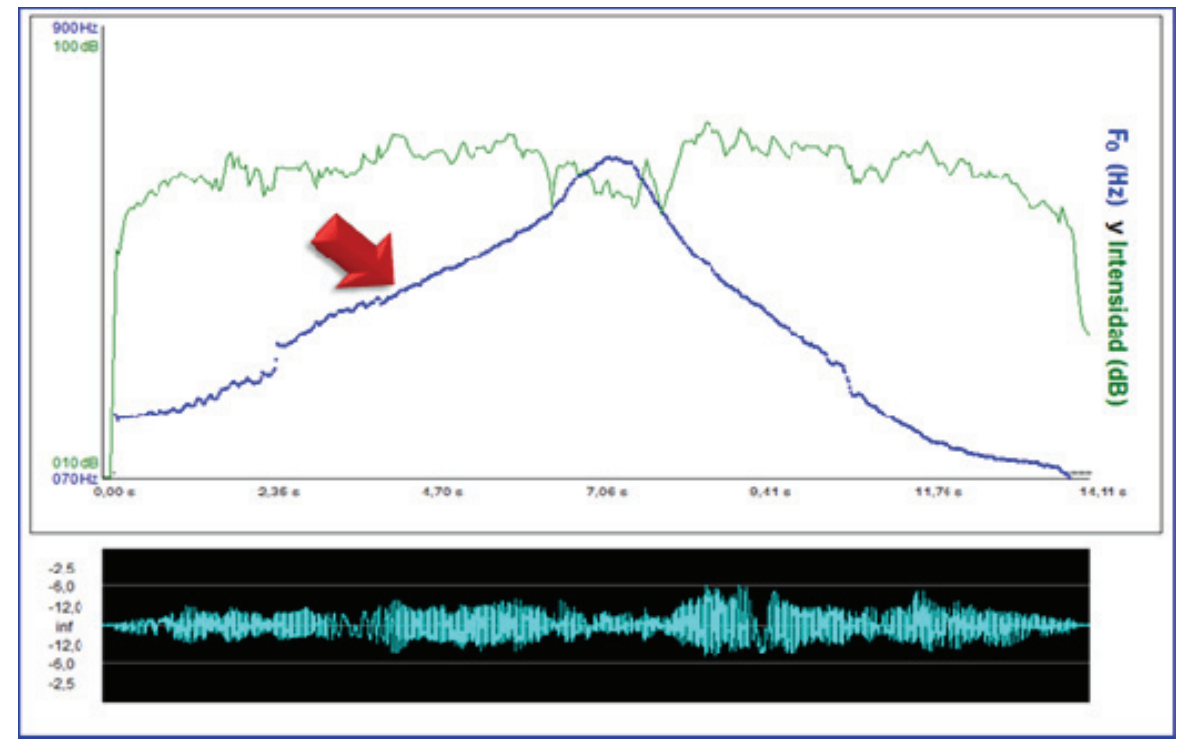

Figura 2 - Diagrama de fonación en tubo produciendo glissandos ascendentes y descendentes 
3) Fonación en tubo haciendo acentos de intensidad y frecuencia con utilización de la musculatura abdominal. La realización de los acentos fue en forma ascendente. Figura 3

4) Producción de la melodía de la canción "Cumpleaños Feliz" dentro del tubo. Figura 4

La duración de la secuencia completa de ejercicios fue de 10 minutos. Cada tarea fonatoria se realizó durante 2.5 minutos. Se permitió que los pacientes pudieran inspirar las veces que fuera necesario durante la realización de cada ejercicio. Después de la secuencia y antes de realizar la grabación post-terapia, se solicitó a los sujetos completar un protocolo de autovaloración vocal modificado, obtenido de un estudio anterior ${ }^{15}$. Este protocolo incluye tres tipos de preguntas: cambios en la voz, sensaciones posteriores a los ejercicios y evaluación general del efecto de los ejercicios.

El artículo fue aprobado por la comisión de ética de investigación de la instituicion de origen con el número 21/2010.

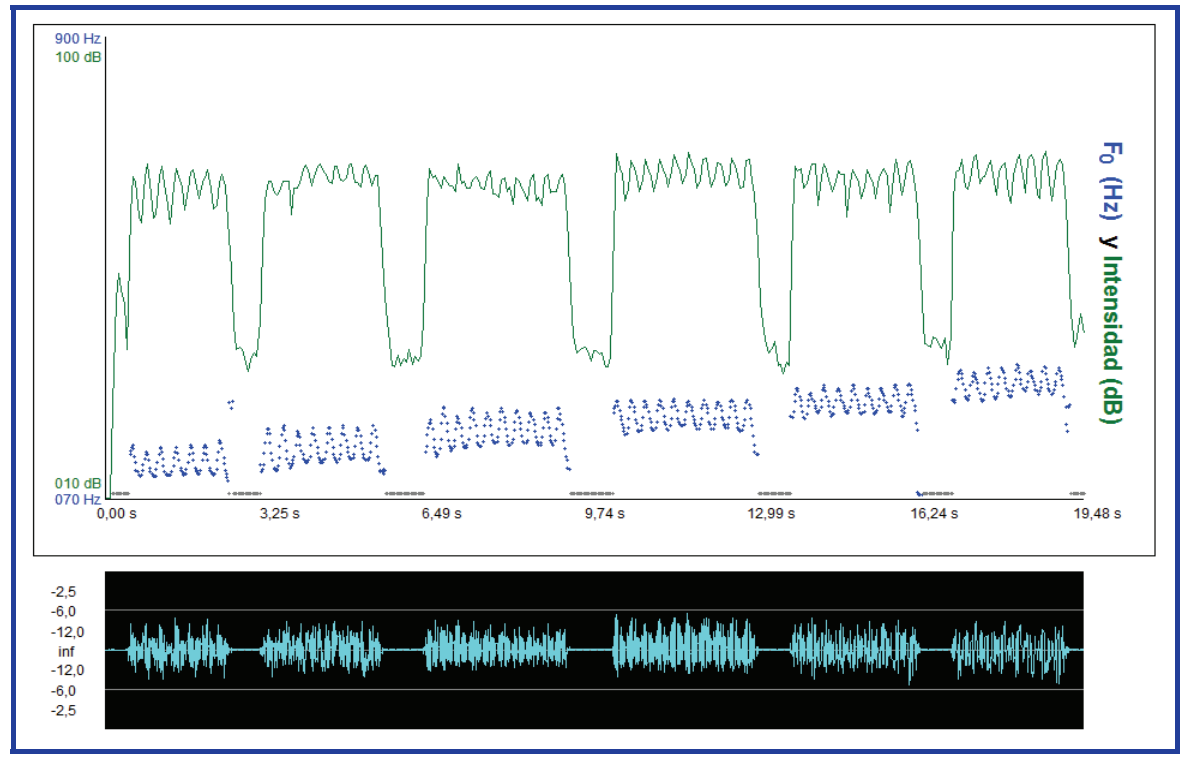

Figura 3 - Diagrama de fonación en tubo haciendo acentos de intensidad y frecuencia con utilización de la musculatura abdominal. Realización de los acentos en forma ascendente

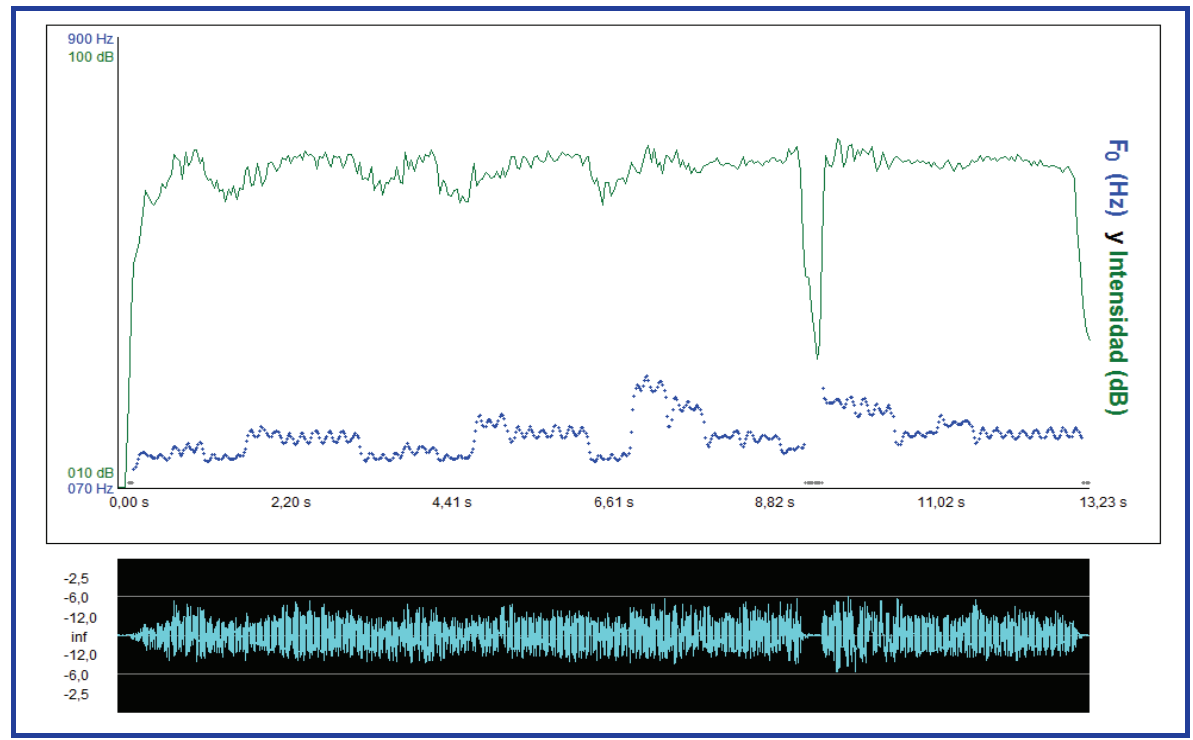

Figura 4 - Diagrama de la producción de la melodía de la canción “Cumpleaños Feliz" dentro del tubo 


\section{Análisis estadístico}

Los datos fueron analizados mediante el programa estadístico STATA 10.1 (StataCorp. 2008. College Station, TX: StataCorp LP.) Los parámetros acústicos de la voz son expresados en media y desviación estándar. Para comparar los valores pre y post tratamiento se utilizó la prueba t de Student. La correlación del cambio de los valores de los parámetros acústicos posterior al tratamiento, se calculó mediante el coeficiente de correlación de Pearson (r) pareado, entre Cesptrum-NHR y Jitter-Shimmer, basados en la suposición de que los primeros suben y los segundos bajan después de la secuencia de ejercicios vocales aplicada. Para este cálculo se utilizó la diferencia pre y post tratamiento con el objetivo de incorporar al modelo la influencia de éste. En toda dócima de hipótesis se consideró un valor $p<0.05$ como estadísticamente significativo.

\section{RESULTADOS}

En la tabla número 1 se muestran los parámetros acústicos de la voz expresados en media y desviación estándar. Las diferencias pre y post tratamiento de todos los parámetros analizados en este estudio (Cepstrum, Jitter, Shimmer y NHR) fueron estadísticamente significativas. Los parámetros con mayor significancia estadística fueron Cepstrum y NHR, ambos con p-value $<0.0001$.

Tabla 1 - Promedio y desviacion estandar de los parámetros acústicos Cepstrum, Jitter, Shimmer y NHR

\begin{tabular}{lccc}
\hline Parámetro & $\begin{array}{c}\text { Pre-tratamiento } \\
\text { (media } \pm \text { DE) }\end{array}$ & $\begin{array}{c}\text { Post-tratamiento } \\
\text { (media } \pm \text { DE) }\end{array}$ & p-value \\
\hline Cepstrum & $2100.84 \pm 437.04$ & $2949.38 \pm 728.30$ & $<0.0001$ \\
\hline Jitter & $0.75 \pm 1.33$ & $0.33 \pm 0.15$ & 0.048 \\
\hline Shimmer & $4.76 \pm 4.66$ & $2.90 \pm 1.32$ & 0.0129 \\
\hline NHR & $19.39 \pm 4.61$ & $22.67 \pm 4.18$ & $<0.0001$ \\
\hline
\end{tabular}

Respecto a la correlación entre los parámetros acústicos, la figura 5 muestra la ccorrelación lineal ajustada entre Cesptrum/NHR, obteniéndose un valor $r=-0.1928$, y $p=0.3559$. La figura 6 grafica la correlación lineal ajustada entre Jitter/Shimmer $r=0.8637, p<0.0001$. Estos datos indican que Jitter y Shimmer disminuyen simultáneamente con un $86 \%$ de correlación, estadísticamente significativa. No así Cesptrum y NHR, que no variaron conjuntamente como se esperaba.

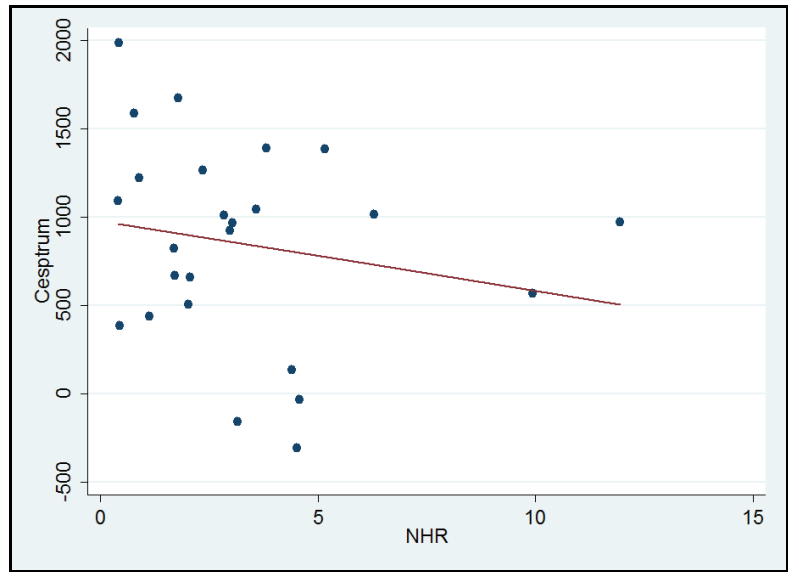

Figura 5 - Correlación lineal ajustada entre Cepstrum y NHR $(r=-0.1928, p=0.3559)$

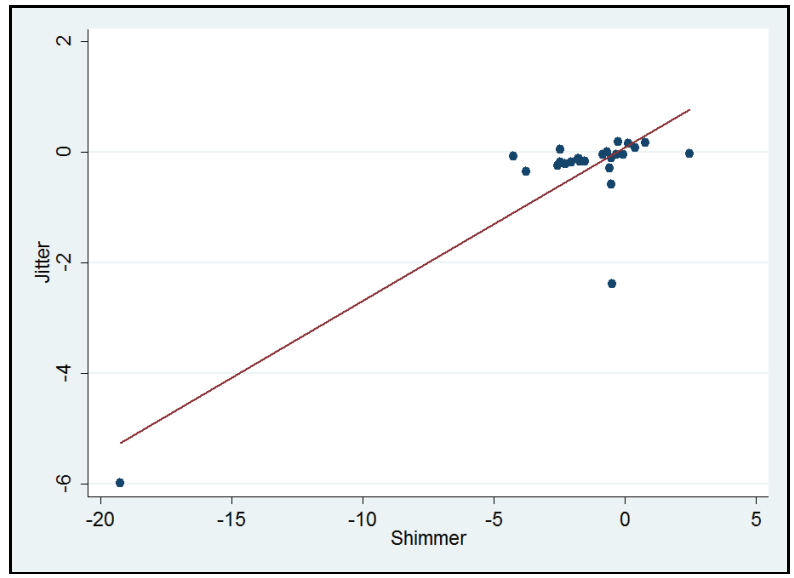

Figura 6 - Correlación lineal ajustada entre Jitter y Shimmer $(r=0.8637, p<0.0001)$ 
La tabla número 2 muestra la distribución numérica y porcentual de las repuestas de autovaloración vocal aplicada inmediatamente posterior al tratamiento. Se observa que los cambios positivos predominan sobre los negativos. En los cambios vocales positivos 16 sujetos $(11.18 \%)$ señalaron que la voz se siente más clara después de los ejercicios; 14 (9.79) señalaron que la voz es de más fácil producción; y 13 (9.09) que la voz se siente más fuerte. Solo 3 sujetos (6.29) señalaron un cambio negativo (voz más débil). Respecto a las sensaciones especificas de la voz, hubo también un predominio de los cambios positivos sobre los negativos. 17 sujetos
(11.88\%) señalaron sentir la voz más estable después de la realización de los ejercicios vocales aplicados, $16(11.18 \%)$ señalaron sentir la garganta más abierta y relajada, $12(8.39 \%)$ músculos más sueltos, y 10 sujetos $(6.69 \%)$ sintieron la voz con colocación más anterior y más proyectada. En este ítem, solo 3 sujetos señalaron sensaciones negativas, $2(1,39 \%)$ de ellos sintieron la voz más inestable y 1 (0.69) la garganta más apretada. Finalmente, en la evaluación general se observa que 22 de los 24 sujetos en estudio opinaron que la voz mejora después de la realización de los ejercicios vocales aplicados.

Tabla 2 - Distribucion numerica y porcentual de las respuestas de autovaloracion vocal

\begin{tabular}{|c|c|c|}
\hline CAMBIOS EN LA VOZ & $\mathbf{N}$ & $\%$ \\
\hline \multicolumn{3}{|l|}{ Cambios Positivos } \\
\hline Voz más fuerte & 13 & 9.09 \\
\hline Voz más clara & 16 & 11.18 \\
\hline Voz mas fácil & 14 & 9.79 \\
\hline \multicolumn{3}{|l|}{ Cambios negatives } \\
\hline Voz más débil & 3 & 2.09 \\
\hline Voz más sucia & 0 & - \\
\hline Voz mas difícil & 0 & - \\
\hline Tono & & - \\
\hline Voz más delgada (aguda) & 9 & 6.29 \\
\hline Voz más gruesa (grave) & 6 & 4.19 \\
\hline \multicolumn{3}{|l|}{ SENSACIONES } \\
\hline \multicolumn{3}{|l|}{ Positivas } \\
\hline Músculos más sueltos & 12 & 8.39 \\
\hline Voz más estable & 17 & 11.88 \\
\hline Garganta más relajada y abierta & 16 & 11.18 \\
\hline Mas vibraciones en la zona anterior (voz adelante, proyectada) & 10 & 6.99 \\
\hline \multicolumn{3}{|l|}{ Negativas } \\
\hline Músculos más tensos & 0 & _ \\
\hline Voz más inestable & 2 & 1.39 \\
\hline Garganta mas apretada & 1 & 0.69 \\
\hline Mas vibraciones en la zona posterior (voz atrás, atrapada) & 0 & - \\
\hline \multicolumn{3}{|l|}{ EVALUACION GENERAL } \\
\hline La voz mejora & 22 & 15.3 \\
\hline La voz empeora & 1 & 0.69 \\
\hline La voz no cambia en nada & 1 & 0.69 \\
\hline Total & 143 & 100 \\
\hline
\end{tabular}




\section{DISCUSIÓN}

El objetivo del presente estudio fue conocer el efecto acústico inmediato de una secuencia de ejercicios fonatorios utilizando tubos de resonancia en un grupo de profesores con voz disfónica (soplada). La secuencia incluyó una fonación vocálica sostenida, glissandos ascendentes y descendentes, acentos de frecuencia e intensidad, y realización de la melodía de una canción. Todas las tareas fonatorias fueron realizadas dentro de un tubo de resonancia.

Al comparar los valores pre y post tratamiento de Cepstrum, Jitter, Shimmer y NHR, se observa una diferencia estadísticamente significativa en todos los parámetros. Esto indica que la secuencia de ejercicios aplicada tiene un efecto positivo en las características vibratorias de los pliegues vocales. En un estudio de similares características, Sampaio et a $\left.\right|^{15}$ utilizando tubos de resonancia y el ejercicio de finger kazoo obtuvieron una diferencia significativa pre y post tratamiento en algunos de los parámetros acústicos analizados. El cambio favorable en los valores obtenidos en forma posterior a la aplicación del tratamiento puede ser atribuido al aumento de la interacción entre la fuente de voz (pliegues vocales) y el filtro (tracto vocal), lo que favorece una fonación más eficiente y económica por el uso de técnicas terapéuticas que involucran semi-oclusión?

Uno de los parámetros que obtuvo mayor significancia estadística fue el Cepstrum. Por considerarse confiable clínicamente, el uso del Cepstrum ha tenido un aumento en los últimos años en la clínica e investigación de la voz ${ }^{16-18}$. El cálculo de este parámetro resulta en la determinación del pico de mayor amplitud en una escala temporal, lo que permite calcular el valor de la F0; y además la amplitud del pico del Cepstrum entrega información del grado de periodicidad de la señal de más baja frecuencia en relación a los otros componentes periódico o de ruido presentes ${ }^{19}$. Mediante el Cepstrum es posible obtener características que miden la calidad de la voz. La riqueza espectral puede cuantificarse por medio de la amplitud y la anchura del componente Cepstral correspondiente al pitch. Si se presenta un pico con amplitud considerable, se está señalando la presencia de energía de dicho componente, siendo una característica de las voces de gran calidad. También una anchura reducida del pico Cepstral indica una alta estabilidad de la frecuencia del pitch ${ }^{20}$. En el presente estudio se consideró el promedio de la amplitud del pico Cepstral en 6 puntos diferentes de la zona media de cada onda analizada. Este promedio resultó ser mayor para las muestras tomadas posterior a la terapia, indicando que la secuencia de ejercicios con tubos de resonancia aplicada produjo un aumento del grado de periodicidad y aumento de la energía de FO. Una voz normal teniendo una estructura armónica bien definida, posee un fuerte pico Cepstral, comparado con una voz soplada y ronca con pobre definición de la estructura armónica que posee un pico Cepstral más débil ${ }^{16}$. De acuerdo a lo señalado, se puede inferir que a pesar que las voces continuaron siendo disfónicas después de la terapia, se produjo un aumento de la definición de la estructura armónica reflejado en el aumento del valor del pico del Cepstrum. En la figura 7 se muestran a modo de ejemplo dos picos del Cepstrum de un mismo paciente tomados desde la muestra pre y post tratamiento.

Otro parámetro acústico que fue sensible al cambio vocal en este estudio, fue el índice armónico/ruido (NHR). Las voces sopladas tienen como característica el reemplazo de armónicos por ruido en la señal acústica. Normalmente, las voces presentan dos componentes principales: ondas periódicas y ruido al azar ${ }^{21}$. En las voces roncas el primer componente es contaminado por el segundo. Este grado de contaminación puede ser expresado por el índice NHR. En la evaluación pre tratamiento, se obtuvieron valores menores en comparación con la medición posterior. Este cambio indicaría que los ejercicios con tracto vocal semi-olcuido aplicados mejoraron el grado de aducción de los pliegues vocales durante la fonación de una vocal mantenida y de esta forma aumentó la cantidad de componente armónico y disminuyó el componente de ruido (característico de las voces de mejor calidad). Este cambio puede ser explicado fisiológicamente no solo por el aumento del cociente de aducción de los pliegues vocales, sino que también por el aumento el skeewing o inclinación de la onda del pulso del flujo glotal, lo que sugiere un tiempo de cierre más rápido de los pliegues vocales en relación al tiempo de apertura producido por el uso de posturas semiocluidas del tracto vocal ${ }^{10}$. Este hecho facilitaría la fonación y produciría un fortalecimiento de los armónicos más altos y un incremento del nivel de presión sonora ${ }^{13}$. Estos cambios fisiológicos y acústicos contribuyen a la producción de una voz perceptualmente más resonante y brillante. Al respecto, Verdolini-Marskon ${ }^{22}$ y Verdolini ${ }^{23}$ mostraron que la voz resonante requiere una posición de los pliegues vocales casi abducida o casi aducida, señalando además que los ajustes supraglóticos contribuyen a esta configuración glotal y por lo tanto al realce del espectro glótico. A través de estos eventos fisiológicos y acústicos se puede afirmar que la voz puede ser producida de una forma más económica y eficiente. Titze señala que esta economía fonatoria se produciría por el aumento de la impedancia de entrada al tracto vocal la cual afectaría la forma 


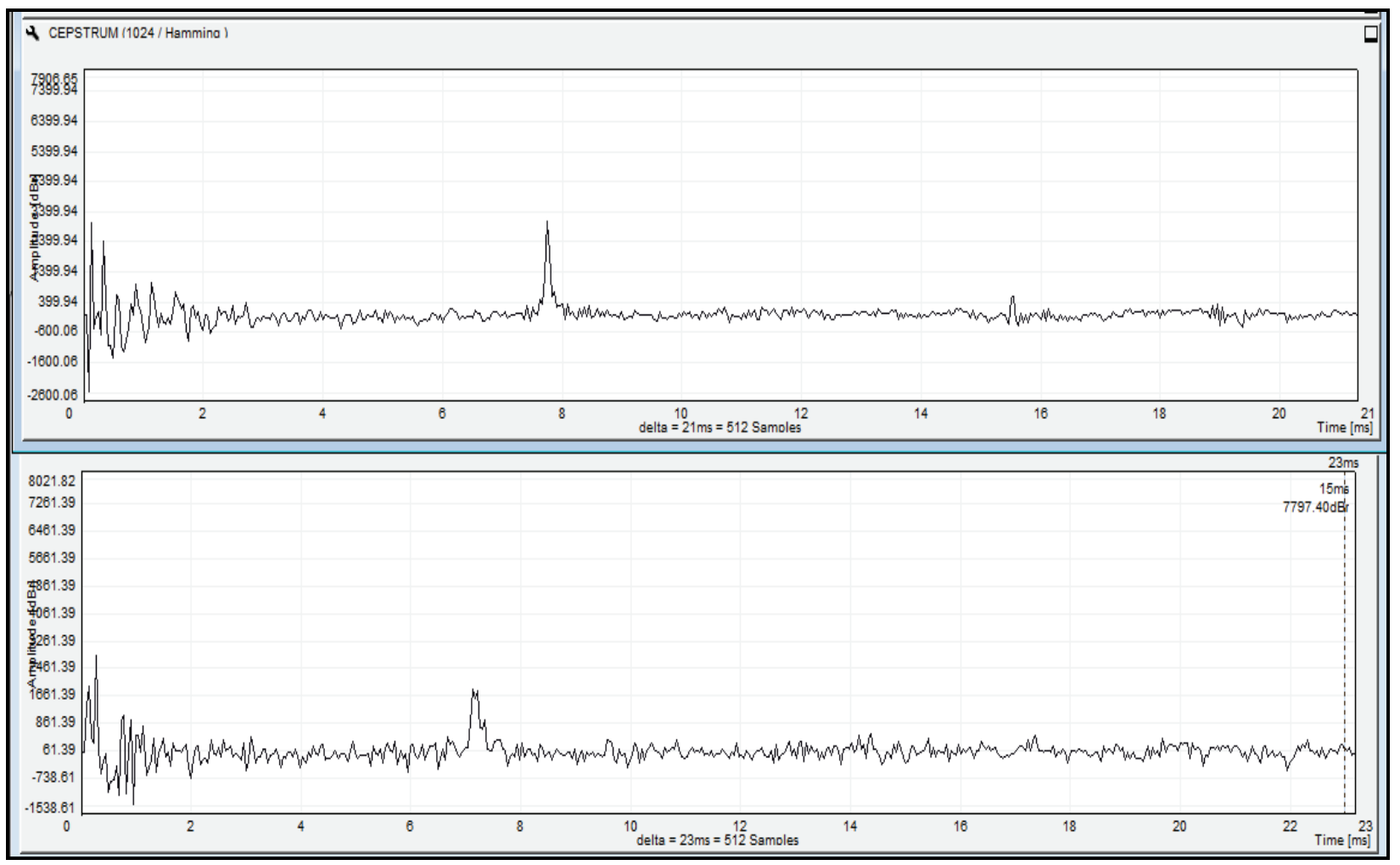

Figura 7 - Picos del Cepstrum de un mismo paciente, tomados desde la muestra pre-tratamiento (abajo) y post-tratamiento (arriba)

del pulso del flujo glotal y modificaría las características oscilatorias de los pliegues vocales ${ }^{24}$. A pesar de haberse obtenido un aumento significativo en los valores de Cepstrum y NHR, no observó una correlación estadísticamente significativa entre estos dos parámetros, hecho que indica que el Cepstrum y el $N H R$ no varían conjuntamente.

Los valores de Jitter y Shimmer descendieron. Este descenso fue estadísticamente significativo, indicando de esta forma que los ejercicios de fonación en tubos de resonancia utilizados en este estudio produjeron un descenso de la perturbación de la frecuencia y amplitud respectivamente. En un estudio realizado con actores portadores de voces normales ${ }^{25}$, se obtuvo una disminución de los valores de Jitter y Shimmer como efecto inmediato después del entrenamiento vocal con el ejercicio de $Y$-buzz. Sin embargo, solo el segundo índice fue estadísticamente significativo. En este mismo estudio, además se obtuvo una disminución significativa de irregularidad de la emisión en la medición post-entrenamiento. En el presente estudio se observo una correlación lineal ajustada entre Jitter/ Shimmer $r=0.8637, p<0.0001$. Estos datos indican que Jitter y Shimmer disminuyen simultáneamente con un $86 \%$ de correlación, estadísticamente significativa.
Los resultados de la encuesta de autovaloración vocal muestran un claro efecto positivo subjetivo de los ejercicios de fonación en tubos de resonancia. En la evaluación general de la secuencia aplicada, 22 de los 24 sujetos señalaron que la voz mejora en forma inmediatamente posterior. En relación a los cambios específicos, se señaló en un alto porcentaje que la voz se siente más fácil y más fuerte durante su producción. En relación a las sensaciones percibidas por los sujetos, las más destacadas fueron: mayor estabilidad vocal, sensación de garganta abierta y músculos más relajados al producir voz inmediatamente después de la secuencia de ejercicios aplicada. Estos resultados sugieren una fonación más sana, probablemente debido al cambio del patrón vibratorio de los pliegues vocales y de la disminución del umbral de presión de la fonación. Este último parámetro es definido como la presión mínima para iniciar la fonación y está relacionado con el estado de salud de los pliegues vocales. Al respecto, Titze ${ }^{24}$ señala que con el uso de ejercicios de tracto vocal semiocluido se produce un descenso del umbral de presión de fonación, lo que causa una producción vocal más económica. Esta economía fonatoria se produciría por el aumento de la impedancia de entrada al tracto vocal la cual afecta la forma del 
pulso del flujo glotal y modifica las características oscilatorias de los pliegues vocales. Por otra parte, la sensación de garganta más abierta y músculos más relajados experimentada por los sujetos, se relacionan con lo señalado por Behlau ${ }^{26}$. La autora afirma que una oclusión parcial en la boca promueve una resonancia retro refleja y expansión de toda el área del tracto vocal, la boca y la laringe. Probablemente esta sensación de apertura y relajación interna permanece después de la realización de los ejercicios. Otro efecto subjetivo producido por los ejercicios aplicados fue el aumento de la sensación de voz clara y la sensación de colocación anterior (vibraciones anteriores). Al respecto Laukkanen ${ }^{5}$ observó que cantantes logran una emisión más clara, brillante y resonante después del uso de tubos de resonancia. Este aumento de la sensación de voz más resonante puede ser explicada por una conversión más eficiente de energía aerodinámica en energía acústica, hecho que produciría un aumento en las sensaciones de vibración y colocación anterior de la voz. Al respecto, Titze ${ }^{6}$ propone que la combinación de un tracto vocal inertivo y la configuración casi aducida/casi abducida de los pliegues vocales es responsable de la máxima transferencia de energía desde la glotis a los labios. Este incremento de la inertancia produce una vibración de los pliegues vocales más fácil. En dos estudios ${ }^{25}$.
${ }^{27}$ donde se investigó el efecto perceptual y acústico del entrenamiento vocal con $Y$-Buzz (ejercicio considerado con tracto vocal semi-ocluido) en actores de teatro, se observó que la emisión de la vocal /i/ pre-entrenamiento es más resonante que la misma emisión anterior al entrenamiento. La valoración de voz resonante en este estudio se realizó mediante el uso de jueces externos a la investigación, los cuales valoraron perceptualmente las voces pre y post entrenamiento. Este hecho señala que la voz resonante no solo produce aumento de las sensaciones de vibración en la zona anterior de la cara, sino que también un cambio que se evidencia en forma perceptual-auditiva.

\section{CONCLUSIÓN}

El uso de tareas fonatorias dentro de tubos de resonancia tiene un efecto fisiológico terapéutico inmediato en sujetos con voces disfónicas, perceptualmente clasificadas como sopladas. Este efecto se ve reflejado en el cambio de parámetros acústicos, la percepción subjetiva de mejoría y facilidad en la producción de la voz obtenida posterior a la terapia. Esto se explicaría por el cambio de patrón vibratorio de los pliegues vocales causado por la mayor interacción fuente-filtro al hacer uso de tubos de resonancia.

\begin{abstract}
Purpose: the purpose of this study is to determine the immediately acoustic effect of phonatory exercise sequence using resonance tubes in a group of dysphonic subjects Method: a unique sequence of four phonatory tasks within resonance tubes was applied in twenty-four teachers with dysphonic voices, assessed perceptually with GRBAS scale. Voice samples were recorded before and after the exercise sequence. Acoustic analysis included the following items: Cepstrum, Jitter, Shimmer, noise-harmonic ratio (NHR). Subjects were asked to fill out a vocal self-assessment protocol after the exercise sequence in order to assess the subjective effects of these exercises. Acoustic parameters of voice samples pre and post training were compared. Results: significant changes were obtained in the Cepstral analysis, jitter and harmonic-to-noise ratio. In the self-assessment, positive effects prevailed over the negative ones, being the main, as follows: more steady voice, more clear voice and more open throat sensation. Conclusion: the outcome indicates that the use of phonatory tasks with resonance tubes may have immediate therapeutic physiological effects on subjects with hoarse voices and a subjective perception of improvement in voice production after the therapy.
\end{abstract}

KEYWORDS: Voice; Voice Training; Speech Acoustics

\section{REFERENCIAS}

1. Nix J, Simpson C. Semi-occluded vocal tract postures and their applications in the singing voice studio. J Singing. 2008;64(3):339-42.
2. Titze I, Laukkanen A. Can vocal economy in phonation be increased with an artificially lengthened vocal tract? A computer modeling study. Logoped Phoniatr Vocol. 2007;32(4):147-56. 
3. Cecconello L. Ejercicios de tracto vocal semiocluido, XII Jornadas Foniátricas, Universidad Nacional de San Luis, Facultad de Ciencias Humanas, San Luis, Argentina, 2009, CD ROM, ISBN 978-987-1595-02-0.

4. Simberg S, Laine A. The resonance tube method in voice therapy: Description and practical Implementations. Logop Phon Vocology. 2007;32(4):165-70.

5. Laukkanen A, Titze I, Hoffman H, Finnegan E. Effects of a Semioccluded Vocal Tract on Laryngeal Muscle Activity and Glottal Adduction in a Single Female Subject. Folia Phoniatr Logop. 2008;60(6):298-311.

6. Titze I, Finnegan E, Laukkanen A, Jaiswal S. Raising lung pressure and pitch in vocal warm-ups: the use of flow-resistant straws. J Singing. 2002;58(4):329-38.

7. Simberg S. Prevalence of vocal symptoms and voice disorders among teacher students and teachers and a model of early intervention. Finland: University of Helsinki, Department of Speech Sciences; 2004

8. Laukkanen A, Pulakka H, Alku P, Vilkman E, Hertegård S, Lindestad PA, Larsson $\mathrm{H}$, Granqvist S. High-speed registration of phonation-related glottal area variation during artificial lengthening of the vocal tract. Logoped Phoniatr Vocol. 2007;32(4):157-64.

9. Titze I. Voice training and therapy with a semioccluded vocal tract: rationale and scientific underpinnings. J Speech Lang Hear Res. 2006; 49(2):448-59.

10. Story B, Laukkanen A, Titze I. Acoustic impedance of an artificially lengthened and constricted vocal tract. J Voice. 2000;14(4):455-69. 11. Rothenberg M. Source-tract acoustic interaction and voice quality. In: Lawrence VL, editor. Transcripts of the 12th Symposium Care of Professional Voice, Part I. New York, NY: The Voice Foundation; 1983. p. 25-31.

12. Gaskill C. Erickson, M. 2010. The effect of an artificially lengthened vocal tract on estimated glottal contact quotient in untrained male voices. J Voice, 24, 57-71.

http://dx.doi.org/10.1590/S1516-18462011005000127

RECEBIDO EM: 18/01/2011

ACEITO EM: 06/04/2011

Contacto para correspondencia:

Marco Guzmán

Avenida Independencia 1027, Independencia

Santiago, Chile

E-mail: guzmanvoz@gmail.com
13. Titze I, Story B. Acoustic interactions of the voice source with the lower vocal tract. J Acoust Soc Am. 1997;101(4):2234-43.

14. Laukkanen AM. About the so called "resonance tubes" used in Finnish voice training practice. An electroglottographic and acoustic investigation on the effects of this method on the voice quality of subjects with normal voice. Scandinavian Journal of Logopedics and Phoniatrics. 1992;17(4):151-61.

15. Sampaio M, Oliveira G, Behlau M. Investigation of immediate effects of two semi-ocluded vocal tract exercises. Pro-Fono, revista de actualización científica. 2008; 20(4): 261-6.

16. Kumar B, Bhat J, Prasad N. Cepstral analysis of voice in persons withn vocal nodules. $\mathrm{J}$ Voice. 2009;24(6):651-3.

17. Kumar R, Bhat J, Fahim S, Raju R. Cepstral analysis of voice in unilateral adductor vocal fold palsy. J Voice. In press. 2010.

18. Lowell S, Colton Raymond, Kelley R, Hanhn Y. Spectral-and cepstral-based measures during continuous speech: Capasity to distinguish dysphonia and consistency within a speaker. J Voice. In press. 2010

19. Cecconello L. Farias P, Gurlekian J. Aplicación del Cepstrum en la clínica vocal. Revista de de la Federación de Sociedades de Otorrinolaringologia. 2008;15 (1):12-4.

20. Alonso J, Ferrer M, Leon J, Travieso C. Cuantificación de la voz para su evaluación clínica por medio del análisis acústico. IV Jornadas de Tecnologia del habla. Zaragoza; 8 al 10 de noviembre de 2006.

21. Baken R, Orlikoff R. Clinical measurement of speech and voice. United states of America: Thomson Delmar Learning; 2000.

22. Verdolini-Marskon K, Burke MK, Lesac A, Glaze L, Caldwell E. Preliminary study of two method of treatment for laryngeal nodules. $\mathrm{J}$ Voice. 1995;9(1):74-85.

23. Verdolini K, Druker D, Palmer $P$, et al. Laryngeal adduction in resonant voice. $\mathrm{J}$ Voice. 1998;12(3):315-27.

24. Titze I. Nonlinear source-filter coupling in phonation: Theory. J Acoust Soc Am. 2008; 123(5):2733-49.

25. Barrichelo-Lindström V, Behlau M. Perceptual Identification and Acoustic Measures of the Resonant Voice Based on "Lessac's Y-Buzz"-A Preliminary Study With Actors. J Voice. 2007;21(1):46-53.

26. Behlau M. Voz, o libro do especialista, Vol II. Rio de Janeiro: Revinter; 2005.

27. Barrichelo-Lindström V, Behlau M. Resonant voice in acting students: perceptual and acoustic correlates of the trained Y-Buzz by Lessac. J Voice. 2009; 23(5):603-9. 